\title{
Solid structures generated by capillary instability in thin liquid films
}

\author{
J. Marthelot, ${ }^{1}$ E. F. Strong, ${ }^{2}$ P. M. Reis, ${ }^{3}$ and P.-T. Brun ${ }^{1}$ \\ ${ }^{1}$ Department of Chemical and Biological Engineering, Princeton University, \\ Princeton, New Jersey 08540, USA \\ ${ }^{2}$ Department of Mechanical Engineering, Massachusetts Institute of Technology, \\ Cambridge, Massachusetts 02139, USA \\ ${ }^{3}$ Flexible Structures Laboratory, Ecole Polytechnique Federale de Lausanne, Lausanne 1015, Switzerland
}

(Received 20 February 2018; published 17 October 2018)

\begin{abstract}
This paper is associated with a poster winner of a 2017 APS/DFD Milton van Dyke Award for work presented at the DFD Gallery of Fluid Motion. The original poster is available from the Gallery of Fluid Motion, https://doi.org/10.1103/APS.DFD.2017.GFM.P0018
\end{abstract}

DOI: 10.1103/PhysRevFluids.3.100506

Patterns are ubiquitous in nature, ranging across myriad examples such as the hexagons in dry mud cracks, the arrangement of seeds in plants, the ripples forming on dirt roads, and our fingerprints [1]. Patterns have fascinated generations of scientists from mathematicians to biologists, who have studied the genesis of their shape and arrangement [2,3]. In particular, it has been shown that instabilities are a robust road to pattern formation $[1,4]$. An instability typically has a range of unstable periodical modes, yet the most unstable mode is often the only one observed in experiments. Its growth surpasses all other modes, thereby enforcing the regularity of the outcome of the instability. Here we take

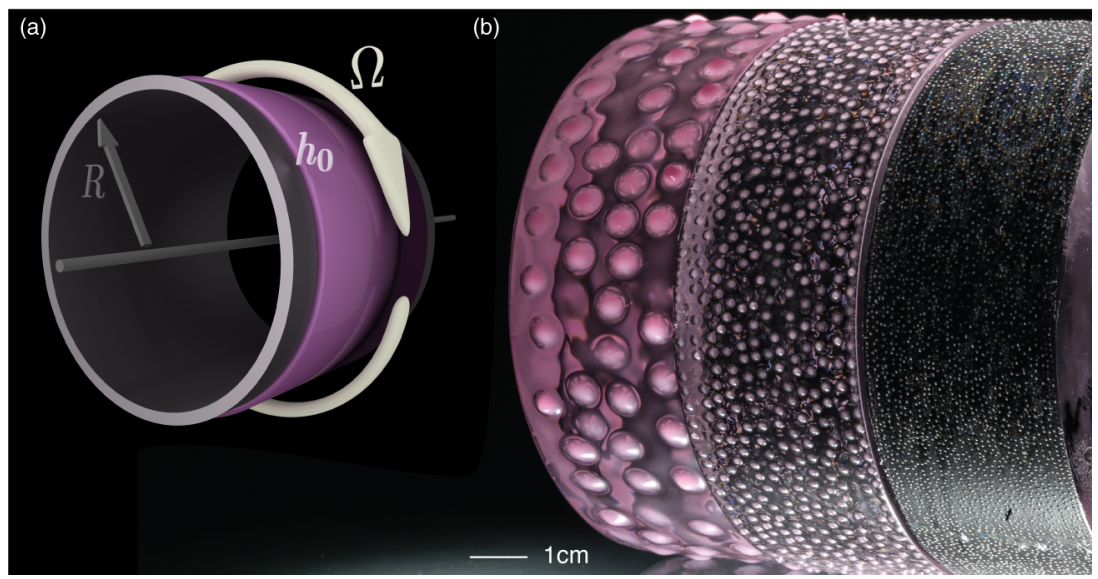

FIG. 1. (a) A thin polymeric film is coated on the outside of a cylinder with radius $R$, which is subsequently rotated at speed $\Omega$. (b) The coating destabilizes to the Rayleigh-Taylor instability and yields drops, whose size decreases with $\Omega R^{2}(4 g, 50 g, 280 g$ from left to right, where $g$ is the acceleration of gravity).

Published by the American Physical Society under the terms of the Creative Commons Attribution 4.0 International license. Further distribution of this work must maintain attribution to the author(s) and the published article's title, journal citation, and DOI. 
(a)

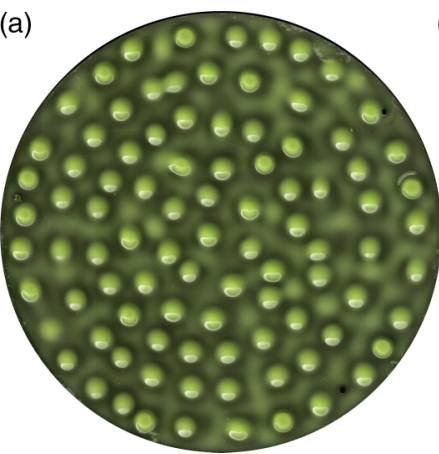

(b)

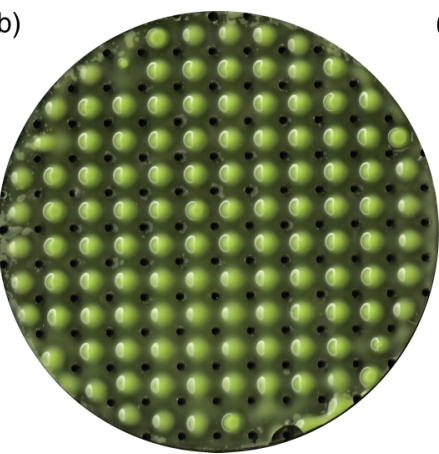

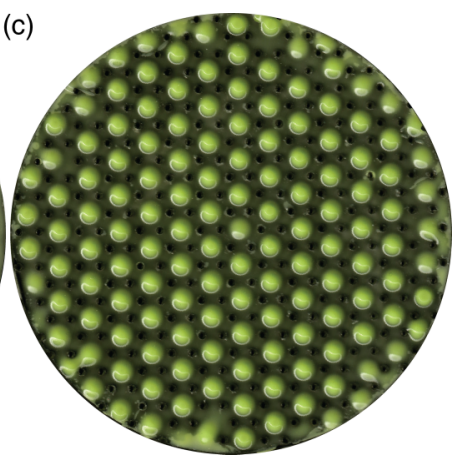

FIG. 2. Taming the instability. (a) Pattern naturally arising in our system. [(b), (c)] Results obtained with seeds arranged in square and hexagonal lattices, respectively. The seeds are drilled into the base plate as evidenced by the small black dots between adjacent drops. Their spacing is $12 \mathrm{~mm}$ so as to match the wavelength of the most unstable mode.

advantage of this fundamental feature and use the Rayleigh-Taylor instability [5] in thin polymeric liquid films to form regular elastic structures [6].

In Fig. 1(a), we show our experimental approach. A thin liquid film is initially deposited on the outside of a cylinder, which is then rotated. As a result, the coating destabilizes due to the Rayleigh-Taylor instability, forming drop lattices with a wavelength set by the balance between capillary forces and centrifugal forces. In particular, as the acceleration increases, the drops become smaller, as evident from Fig. 1(b). As the film cures [7], these drops formed by a fluidic instability eventually yield elastic structures.

In Fig. 2, we show that the instability can be tamed. We have designed defects on the solid substrate onto which the film is initially coated so as to control the organization of the drop arrangement. Square and hexagonal lattices are shown in Figs. 2(b) and 2(c), respectively. In such cases, the amplitude of the drops is almost monodisperse. With this methodology, we have shown that tangible structures can be formed in the laboratory when harnessing an interfacial fluidic instability. In addition to their aesthetically pleasing appearance, these organized drops could find use in practical settings [8-10].

[1] F. Gallaire and P.-T. Brun, Fluid dynamic instabilities: Theory and application to pattern forming in complex media, Philos. Trans. R. Soc. A 375, 20160155 (2017).

[2] D. W. Thompson, On Growth and Form (Cambridge University Press, 1917).

[3] A. M. Turing, The chemical basis of morphogenesis, Philos. Trans. R. London B 237, 37 (1952).

[4] M. C. Cross and P. C. Hohenberg, Pattern formation outside of equilibrium, Rev. Mod. Phys. 65, 851 (1993).

[5] M. Fermigier, L. Limat, J. E. Wesfreid, P. Boudinet, and C. Quilliet, Two-dimensional patterns in RayleighTaylor instability of a thin layer, J. Fluid Mech. 236, 349 (1992).

[6] J. Marthelot, E. F. Strong, P. M. Reis, and P.-T. Brun, Designing soft materials with interfacial instabilities in liquid films, Nat. Commun., doi:10.1038/s41467-018-06984-7.

[7] A. Lee, P.-T. Brun, J. Marthelot, G. Balestra, F. Gallaire, and P. M. Reis, Fabrication of slender elastic shells by the coating of curved surfaces, Nat. Commun. 7, 11155 (2016).

[8] M. Kolle, P. M. Salgard-Cunha, M. R. J. Scherer, F. Huang, P. Vukusic, S. Mahajan, J. J. Baumberg, and U. Steiner, Mimicking the colourful wing scale structure of the Papilio blumei butterfly, Nat. Nanotech. 5, 511 (2010).

[9] J. Shim, C. Perdigou, E. R. Chen, K. Bertoldi, and P. M. Reis, Buckling-induced encapsulation of structured elastic shells under pressure, Proc. Natl. Acad. Sci. U.S.A. 109, 5978 (2012).

[10] J. B. Kim, P. Kim, N. C. Pégard, S. J. Oh, C. R. Kagan, J. W. Fleischer, H. A. Stone, and Y.-L. Loo, Wrinkles and deep folds as photonic structures in photovoltaics, Nat. Photon. 6, 327 (2012). 